\title{
Genetic heterogeneity of Usher syndrome type II
}

\author{
S Pieke Dahl, W J Kimberling, M B Gorin, M D Weston, J M R Furman, A Pikus, \\ C Möller
}

\begin{abstract}
Usher syndrome is an autosomal recessive disorder characterised by retinitis pigmentosa and congenital sensorineural hearing loss. A gene for Usher syndrome type II (USH2) has been localised to chromosome 1q32-q41. DNA from a family with four of seven sibs affected with clinical characteristics of Usher syndrome type II was genotyped using markers spanning the 1q32-1q41 region. These included D1S70 and D1S81, which are believed to flank USH2. Genotypic results and subsequent linkage analysis indicated non-linkage of this family to these markers. The A test analysis for heterogeneity with this family and 32 other Usher type II families was statistically significant at $p<0.05$. Further clinical evaluation of this family was done in light of the linkage results to determine if any phenotypic characteristics would allow for clinical identification of the unlinked type. No clear phenotypic differences were observed; however, this unlinked family may represent a previously unreported subtype of Usher type II characterised by a milder form of retinitis pigmentosa and mild vestibular abnormalities. Heterogeneity of Usher syndrome type II complicates efforts to isolate and clone Usher syndrome genes using linkage analysis and limits the use of DNA markers in early detection of Usher type II.
\end{abstract}

(F Med Genet 1993;30:843-8)

Genetics Department Research Hospital, 555 N 30 Street, Omaha, NE 68131, USA.

$S$ Pieke Dahl

W J Kimberling

M D Weston

Eye and Ear Institute of Pittsburgh,

Pittsburgh, PA, USA.

M B Gorin

J M R Furman

National Institute of

Deafness and other

Communication

Disorders, National

Institutes of Health

Bethesda, MD, USA.

A Pikus

University of

Linkoping, Sweden.

C Möller

Correspondence to Dr Kimberling.

Received 20 January 1993.

Revised version accepted

7 May 1993. is no vestibular dysfunction. The clinical subtypes are consistent in families having more than one affected sib. This suggested genetic heterogeneity, where at least two different mutant genes cause the distinct phenotypes: the severe Usher type I and the milder Usher type II. Usher type II has been localised to chromosome 1q32. ${ }^{12}$ Since Usher type I failed to show the same linkage, ${ }^{1-3}$ it was established that types I and II are not allelic.

Recently, three separate localisations have been reported for Usher syndrome type I: USH1A, USH1B, and USH1C. USH1A has been localised to $14 \mathrm{q}^{4}$ and appears to be restricted to a group of related families from the
Poitou-Charentis region in France. Attempts to reproduce the French results in other families were unsuccessful. ${ }^{5}$ USH1B and USH1C have been localised to the long and short arms of chromosome 11 , respectively ${ }^{67} \mathrm{USH} 1 \mathrm{~B}$ appears to be responsible for the most common variety of Usher type $I$, since mutations at the USH1B locus on $11 \mathrm{q}$ seem to have a wider distribution than the USH1C gene localised to $11 \mathrm{p},{ }^{6}$ and preliminary findings suggest that USH1C may be restricted to French Acadians in Louisiana. ${ }^{7}$ The Poitou-Charentes families failed to show linkage with either $11 \mathrm{q}$ or $11 \mathrm{p}$ markers, thus establishing it as an independent locus for type I Usher syndrome. These discoveries show that heterogeneity of Usher syndrome type $I$ is quite extensive with, as yet, no clinical distinction between subtypes. Here we present a family with the clinical characteristics of Usher syndrome type II which fails to show linkage to DNA markers that bracket the USH 2 gene. This finding indicates the existence of another locus for Usher syndrome type II, and shows that genetic heterogeneity is present in Usher type II as well as Usher type I.

\section{Clinical methods}

SUBJECTS

Families in the Boys Town National Research Hospital (BTNRH) Usher syndrome project have been ascertained and clinically evaluated by methods that have been previously described. ${ }^{89}$ The families in the project are predominantly of northern European extraction. During the course of this study, one family (735) was encountered through self-referral. Detailed records for family 735 were obtained primarily from previous studies done at NIH in 1988. These records were consistent with a diagnosis of Usher syndrome type II. Following genetic analysis at BTNRH, certain family members were re-examined at the Eye and Ear Institute of Pittsburgh in 1991 to verify the clinical diagnosis of Usher syndrome type II.

\section{OPHTHALMOLOGY}

Visual psychophysical testing of family members included visual acuity, contrast sensitivity, colour vision, Goldmann perimetry, and light and dark adaptation studies. Electrophysiological evaluations included an electrooculogram (EOG), and rod-cone responses measured by uniform field, Ganzfeld electroretinography (ERG). The clinical evaluation included intraocular pressures, slit lamp biomicroscopy at the anterior segment and fun- 
dus, and indirect ophthalmoscopy. Fundus changes were documented using standard fundus photography.

\section{AUDIOLOGY}

Audiological evaluations were accomplished in different centres over many years but routinely included traditional measures of pure tone and speech discrimination thresholds, tympanometry, acoustic reflex testing, and, for one patient, auditory brainstem response (ABR) measurement. All audiological testing was accomplished in standard sound isolated testing suites using commercially available calibrated instrumentation.

\section{VESTIBULAR STUDIES}

The vestibular test battery included: (1) an oculomotor screening battery to assess saccades, pursuit, and nystagmus; (2) static positional testing; (3) Hallpike's manoeuvres; (4) alternate binaural bithermal caloric irrigations with $30^{\circ} \mathrm{C}$ and $44^{\circ} \mathrm{C}$ water; (5) computerised dynamic posturography (Equitest); and (6) rotational testing using the sinusoidal harmonic acceleration test.

\section{Laboratory methods \\ SAMPLE COLLECTION}

Blood samples from family 735 were originally collected in 1990. Non-linkage results prompted a repeat sampling which was completed and regenotyped in 1991 to verify that no sample mix up had occurred. Genomic DNA of all families was extracted from lymphocytes in 3 to $5 \mathrm{ml}$ of venous blood or from lymphoblastoid cell lines using an Applied Biosystem 340A nucleic acid extraction machine.

\section{GENOTYPING}

RFLP and STRP genotyping was done according to standard protocols. ${ }^{11}$ DNA markers were chosen based on locus order and genetic distances listed in the CEPH consortium linkage map of chromosome $1 .{ }^{12}$ Recent evidence from the analysis of dinucleotide repeat polymorphisms on 1q indicates that the USH 2 gene lies between D1S70 and D1S81 near PPOL (Weston and Kimberling, unpublished data).

\section{ANALYSIS}

Linkage analyses were performed with genotypic data from 32 type II Usher syndrome families (besides family 735), comprising 252 subjects ( 81 affected). US was assumed to be inherited as a fully penetrant autosomal recessive disorder and was treated as the affected status locus in all analyses. Pairwise and multipoint linkage tests and tests of locus order were performed using the LINKAGE 5.1 program package. Linkage data were then analysed for heterogeneity using the A test computed with the HOMOG computer program. ${ }^{13}$ The loca- tion scores at five equidistant points between D1S70 and D1S81 were used in the A test analysis and $\alpha$, the proportion of unlinked families, was evaluated between 0 and 1.0 at 0.05 intervals.

\section{Clinical results \\ FAMILY HISTORY}

The ancestry of family 735 is predominantly of Irish, Scottish, and Welsh origin. There is no known consanguinity. There is a positive family history of adult onset diabetes in both the maternal and paternal sides. The father reports a mild hearing loss resulting from environmental factors. There are no instances of RP or other hearing loss outside the sibship reported here. Usher syndrome type II was originally suggested as a probable diagnosis when pedigree analysis indicated that the RP was segregating with congenital hearing loss in four of seven sibs (fig 1 shows six of the sibs, insufficient DNA prevented the seventh from being included in the study).

\section{OPHTHALMOLOGY}

All affected sibs reported symptoms of RP from late childhood or early adolescence. In 1988, the parents and affected sibs underwent ophthalmological evaluations at the NIH National Eye Institute. I 8 had no abnormal findings. The fundus of $I \cdot 9$ was normal except for a few macular drusen, which had been noted in previous examinations. Affected sibs II $\cdot 1,5,6$, and 7 displayed a pattern of retinal degeneration that appeared different from RP exhibited in typical Usher syndrome. Clinical examination showed mottling of the retinal pigment epithelium with individual and coalescent greyish $500 \mu$ spots. Despite the evidence of extensive retinal degeneration, few characteristic intraretinal bone spicules pigment deposits were seen. Blood vessels were attenuated, visual fields were constricted, EOGs were subnormal, and ERGs were extinguished or showed thresholds that were extremely depressed in a rod-cone pattern. The diagnosis of 'RP sine pigmento' with hearing loss was made.

In 1991, after DNA testing of family 735 showed non-linkage to markers in the 1q32q41 region, the parents, affected sibs II 5,6 , and 7 , and unaffected sib II.4 were re-examined at the Eye and Ear Institute of Pittsburgh. The father displayed mild bilateral diabetic retinopathy. II 4 was normal. The three affected sibs presented with decreased visual acuities, worsened symptoms of night blindness and tunnel vision, further attenuation of retinal blood vessels, increased mottling of the RPE, but still few intraretinal pigment deposits (fig 2). The general appearance of each fundus resembles an early stage of RP. ${ }^{14}$

\section{AUDIOLOGY}

All affected sibs showed symptoms of congenital hearing loss, verified in early childhood. Patient II 1 , evaluated at the age of 34 , exhib- 


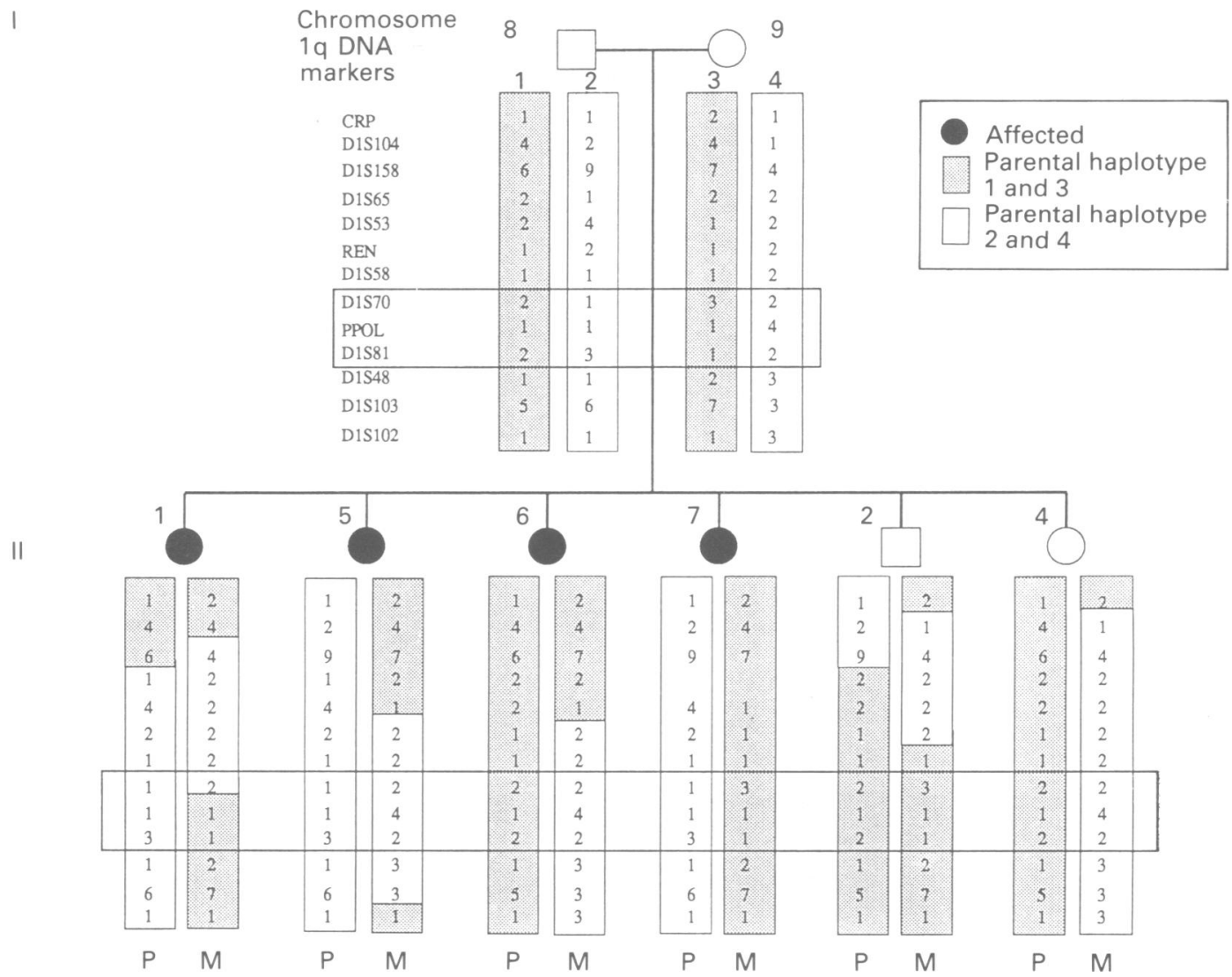

Figure 1 Abbreviated pedigree of family 735 with chromosome 1q DNA marker types of each subject shown underneath, illustrating no consistent segregation of haplotypes with affected status.

ited a bilateral moderate to profound sensorineural hearing loss. During a reassessment two years later, the pattern of hearing loss was essentially unchanged with no progression. Tympanometry was determined to be within normal limits bilaterally, and acoustic reflexes were in agreement with the degree of hearing loss.

II. 5 was evaluated at the age of 27 , approximately three weeks after a car accident in which she suffered concussion. She was found to have bilateral severe to profound, predominantly sensorineural, hearing loss. She had no measurable speech discrimination to the maximum output without visual cues. Results of tympanometry were normal bilaterally. Acoustic reflexes were absent bilaterally, which was consistent with the degree of hearing loss observed.

Patient II. 6 was evaluated at the age of 21 . Her pure tone thresholds showed a sensorineural hearing loss bilaterally, mild at $500 \mathrm{~Hz}$ and moderately severe in the high frequencies. Speech thresholds were consistent with pure tone low frequency responses and indicated a moderate deficit. Acoustic immittance results were within normal range for tympanometry and acoustic reflex studies, bilaterally. Reflex thresholds in the high frequencies were consistent with cochlear hearing loss bilaterally. ABR studies were within normal limits, bilaterally; however, at rapid stimulus rates there was a marked deterioration of right ear responses in comparison with the left.

Patient II.7 underwent audiological examination at the age of 17 , indicating a moderate to severe bilateral symmetrical sensorineural hearing loss. Aided, suprathreshold speech recognition was excellent bilaterally, but speech tolerance level was somewhat reduced. She achieved a speech reception threshold of $22 \mathrm{~dB}$ aided, and speech recognition was excellent without visual cues.

Fig 3 shows an audiogram from each affected sib of family 735 against an observed range calculated using 32 typical Usher type II patients. The hearing of the affected sibs at all frequencies is within $2 \mathrm{SD}$ of the normal Usher II range.

\section{VESTIBULAR TESTING}

The first vestibular test of patient II.5 was done soon after the car accident. At that time, II 5 complained of dizziness and headaches dating from the accident. A right directional preponderance was seen on the rotational test, but the same finding was not observed again when vestibular testing was repeated a year later. The second evaluation did show a left beating nystagmus on static positional testing; however, this could have resulted from the accident.

Patient II 6 reported no vestibular symptoms. Alternate binaural bithermal caloric testing was indicative of a reduced vestibular response on the right ear of $33 \%$, consistent with a mild right peripheral vestibular lesion. Posturography was abnormal in a non-specific pattern; normal with the exception of adaptation to toes down rotation, resulting in abnormal movement coordination.

At the age of 16 , patient II. 7 complained of headaches, dizziness, vomiting, and tinnitus 

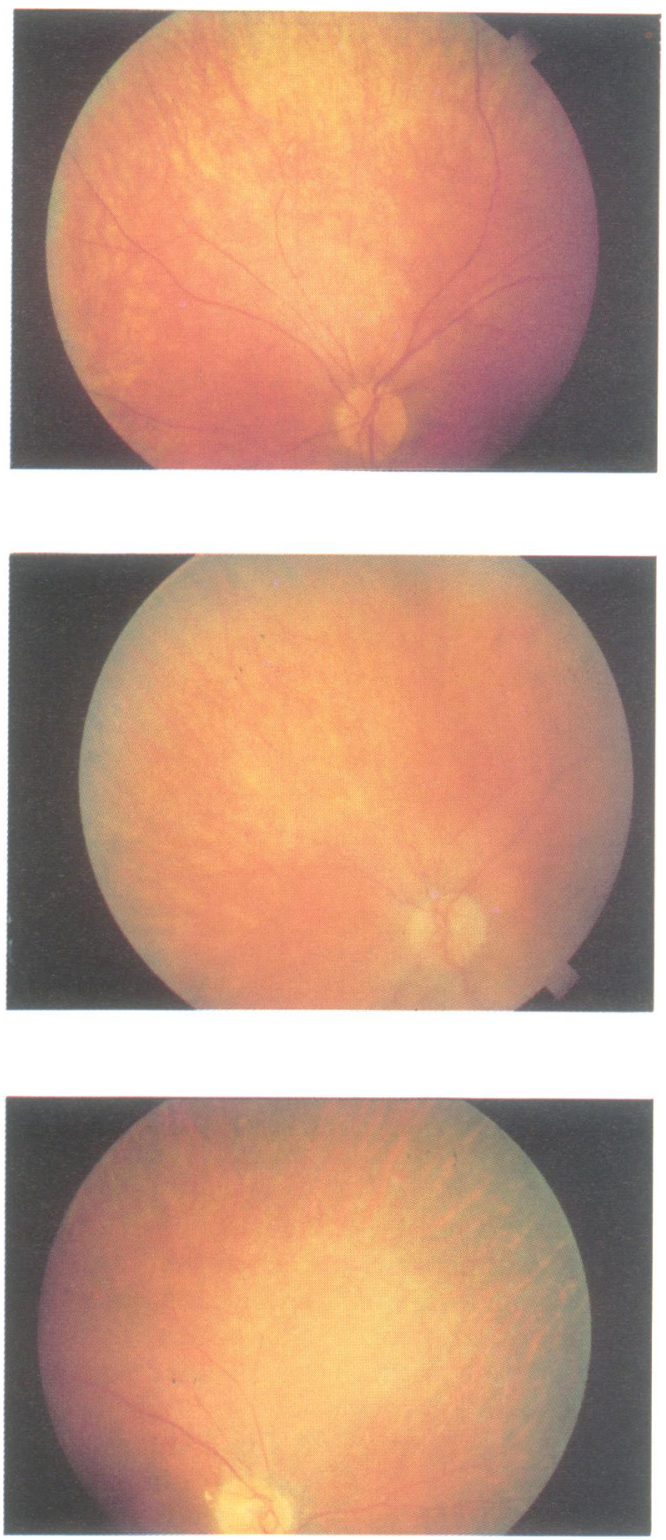

Figure 2 Fundus photographs of three affected sibs from family 735 show relatively few pigment deposits, characteristic of early $R P$.

for intervals that had lasted three weeks. Examination showed no obvious neurological deficit. Neurological examination, magnetic resonance imaging (MRI) of the brain, electroencephalography (EEG), and electronystagraphy (ENG) results were all within normal limits. Patient II.7 was 20 when vestibular testing was repeated and all findings were normal.

\section{Molecular results \\ HAPLOTYPES}

Fig 1 shows the chromosome 1 haplotypes of family 735 in the most likely phases assuming fewest crossovers between markers, after all possible 735 parental phases were considered. Affected status does not cosegregate with the DNA markers D1S70 and D1S81 which flank USH2. For this phase, three double crossovers are necessary if one USH2 gene is placed between D1S70 and D1S81. Assuming the locus order is correct and that double cross- overs are highly improbable, these results are not compatible with linkage to markers on 1q32-1q41. Retyping of family 735 using DNA from the second samples confirmed that the original results were valid and no sample mix up had occurred. No instance of nonpaternity was found in over 30 polymorphisms which have been tested for the family.

\section{LINKAGE ANALYSIS}

The conclusions are further supported by a significantly negative set of multipoint lod scores for family 735 with chromosome 1q markers that span the region between CRP and D1S102. Fig 4 shows the multipoint likelihood distribution for only family 735 compared with that of all 32 Usher II families. The peak lod score of the Usher II sample approaches 15 between markers D1S70 and D1S81. At midway between D1S70 and D1S81, family 735's lod score is less than -2.00 , consistent with the hypothesis that family 735's Usher gene is not in the USH2 region. This contention was further reinforced by Ott's HOMOG A test for heterogeneity, which resulted in a $\chi_{(1)}^{2}=5.255, \quad p<0.05$, supporting heterogeneity. Of the 33 families, only 735 showed a low posterior probability of linkage at 0.02 . The distribution of the posterior probabilities for the remaining families was: 0.71 (one family), 0.80 to 0.90 (six families), and $>0.90$ (25 families). There is no statistically significant evidence of any other unlinked families in this sample. The $\chi^{2}$ was reduced to 0.00 when family 735 was removed from the sample.

\section{Conclusion}

We conclude that family 735 represents a variant of Usher syndrome type II and propose that the locus be designated USH2b when it is identified. The locus on 1q would be USH2A to be compatible with the previous suggestion of USH1A, USH1B, and USH1C for the Usher type I loci. The variant of Usher type II may be distinguished from the common Usher type II by a form of RP with fewer pigment deposits and mild vestibular defects, but these observations must be verified with additional Usher IIb families.

\section{Discussion}

Localisation and isolation of a gene relies on an accurate clinical diagnosis. Clinical characteristics of family 735 did not differ significantly enough to prevent the inclusion of that family into the Usher type II sample for linkage analysis. However, after non-linkage was shown, further examination and re-evaluation of family 735 was warranted to prevent inadvertent inclusion of additional Usher IIb families into the common Usher IIa sample. The affected subjects in family 735 display a variant of RP, namely RP sine pigmento. Heckenlively ${ }^{14}$ outlined the early stages of $R P$ as being characterised by generalised diffuse depigmentation of the RPE, relatively normal retinal vessels, but with an abnormal but not 


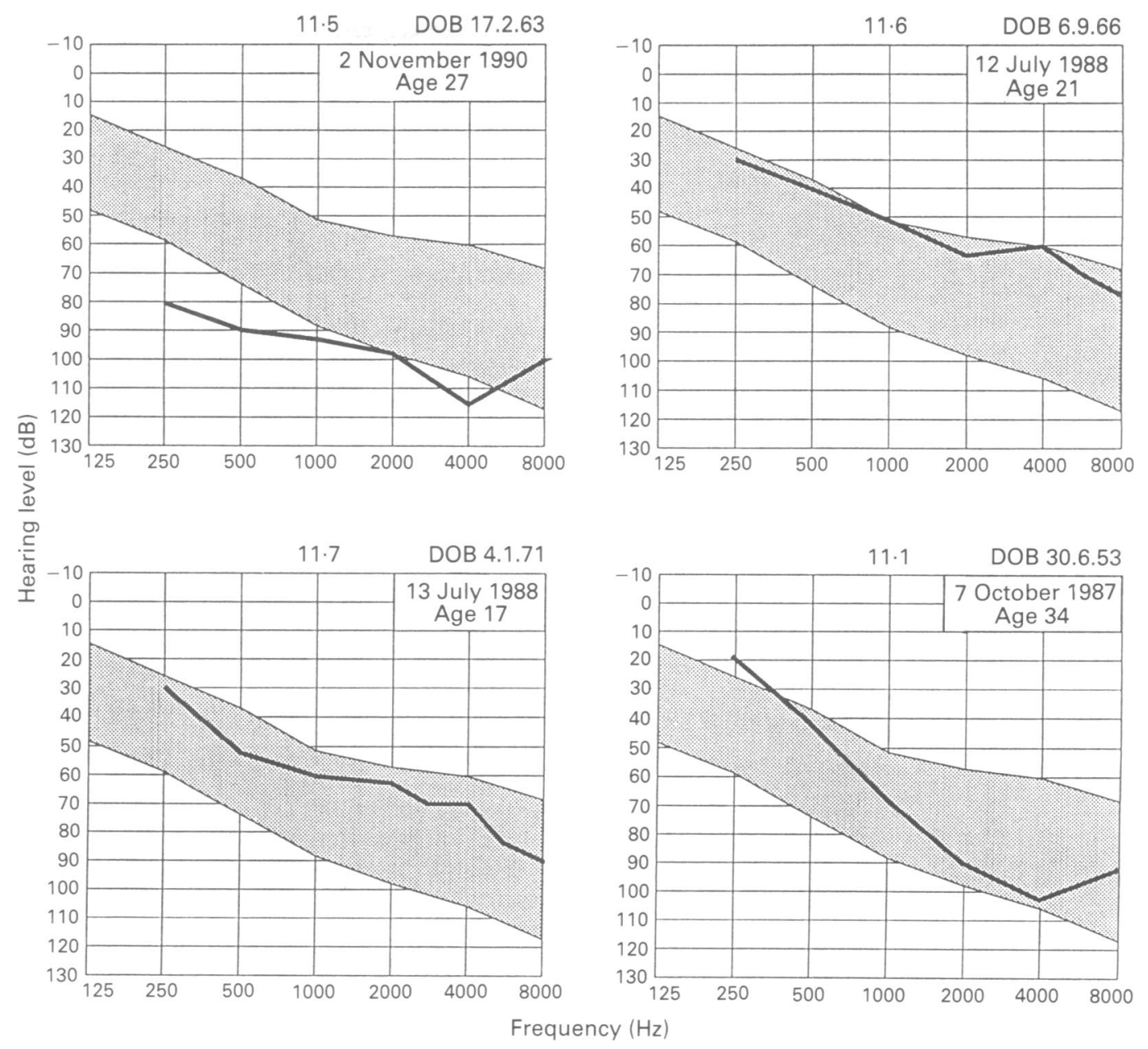
Figure 3 Audiograms of affected sibs from family 735 do not differ significantly from the range seen in typical
Usher syndrome type II (shaded area).

extinguished ERG, night blindness, and somewhat constricted visual fields. Little or no pigment deposition is seen in almost all RP patients initially, and this particular stage is more persistent in some cases. This description is very similar to retinal findings of family 735 (fig 2). Family 735's pattern of hearing loss does not significantly deviate from that of

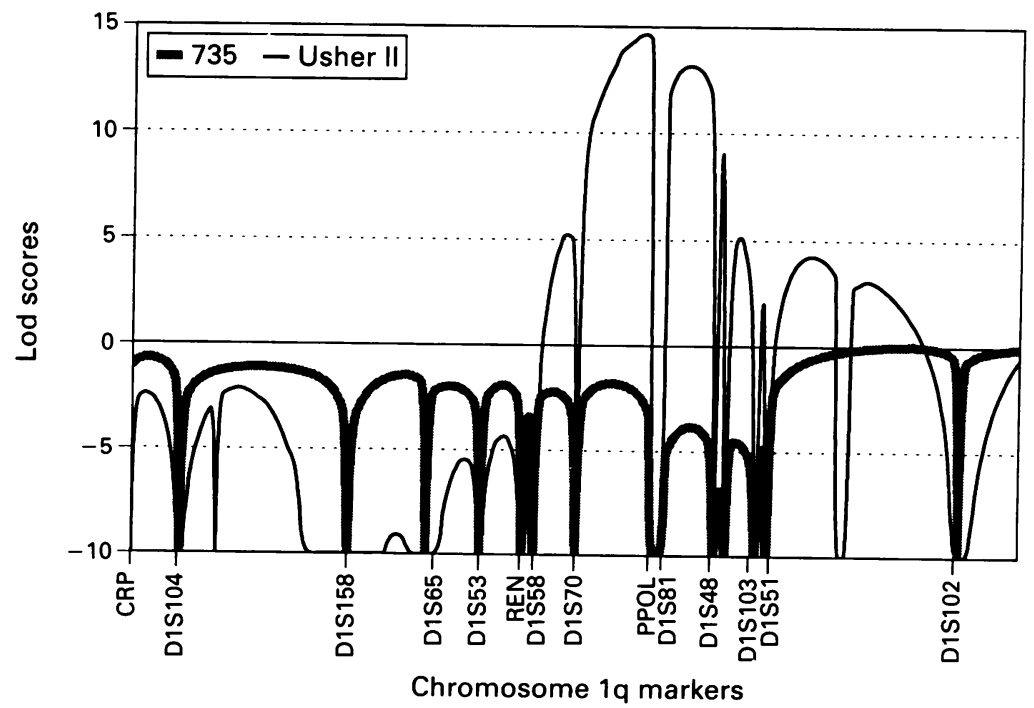

Figure 4 Multipoint analysis of 32 Usher II families typed with chromosome 1 DNA markers displays high probability of linkage (dotted line) versus negative results for
family 735 (solid line). typical Usher II, and similar variation within Usher II families has been observed. The origin of this variation can be found in a variety of environmental or genetic factors unrelated to Usher syndrome, or by the fact that the psychoacoustic test is inherently subject to some variation. If there is a progressive component, it is much less pronounced than reported for proposed subtype Usher type III. ${ }^{15} 16$ Hearing loss appears to have progressed in II.5, but one might postulate that chronic hypothyroidism is somehow involved since she is the only sib showing a progressive loss and hypothyroidism has been implicated in progressive hearing loss. Vestibular abnormalities within Usher syndrome type II have been previously reported. ${ }^{17}$ Mild vestibular abnormalities as displayed by family 735 could be a way to distinguish phenotypically between the two subtypes of Usher II before DNA analysis, if similar defects were consistently observed in other families unlinked to 1q32-41 markers. Vestibular findings are too variable in family 735 to be considered diagnostic without such verification.

Extensive genetic heterogeneity in Usher syndrome has become increasingly apparent as research progresses. With this report, mutations at at least five different loci can cause Usher syndrome: three for type I which have been localised to chromosomes 11p, 11q, and 
$14 \mathrm{q}$, and two for type II, one localised to $1 \mathrm{q}$ and another located somewhere else. It is reasonable to assume these genes are functionally related; some may code for similar functions or code for enzymes in a common pathway vital for normal vision and hearing. The identification and cloning of the Usher genes is necessary to discover the different role each plays.

There are two important implications with regard to these findings. First, the use of genetic markers for prenatal diagnosis of Usher type II will be confounded by heterogeneity. Although the unlinked type is relatively uncommon, a small error, equal to a value no greater than the fraction of unlinked families, will be associated with linkage based predictions. The problem will not be overcome until the specific genes and mutations associated with Usher syndrome are identified. Second, the process of pinpointing the precise segment containing the USH2a gene depends on observation of critical crossovers and false results from inclusion of a variant family in our sample might not be detected. If that family provides what appeared to be a critical crossover, the search for the USH2a gene could be misdirected. Some clinical parameter that allows the subdivision of the sample into Usher II and II variant without regard to marker typing results is required. We are currently expanding our sample, since accumulation of further Usher IIb families may provide a clinical distinction between different Usher II types. Although family 735 is highly informative, other linked Usher II families must be ascertained before the second locus can be found by linkage analysis. After the genes are located, other methods could be used to characterise the causative factors.
This research was supported by grant $\mathrm{P} 50$ DC00215, NIDCD, and grants from the RP, George Gund, and Deafness Research Foundations. We thank $\mathrm{K}$ Brennan and C Connolly for their technical expertise.

1 Kimberling WJ, Weston MD, Moller C, et al. Localization of Usher syndrome type II to chromosome 1q. Genomics 1990;7:245-9.

2 Lewis RA, Otterud B, Stauffer D, Lalouel JM, Leppert M. Mapping recessive ophthalmic diseases: linkage of the locus for Usher syndrome type II to a DNA marker on chromosome 1q. Genomics 1990;7:250-6.

3 Kaplan J, Guasconi G, Bonneau D, et al. Usher syndrome type $I$ is not linked to D1S81 (pTHH 33): evidence for

4 Kaplan J, Gerber S, Bonneau D, et al. A gene for Usher syndrome type I (USH1A) maps to chromosome 14q. Genomics 1992;14:979-87.

5 Keats BJB, Todorov AA, Atwood LD, et al. Linkage studies of Usher syndrome type I: exclusion results from the Usher syndrome consortium. Genomics 1992;14:70714.

6 Kimberling WJ, Moller CG, Davenport S, et al. Linkage of Usher syndrome type I gene (USH1B) to the long arm of chromosome 11. Genomics 1992;14:988-94.

7 Smith RJH, Lee EC, Kimberling WJ, et al. Localization of two genes for Usher syndrome type I to chromosome 11. Genomics 1992;14:995-1002.

8 Kimberling WJ, Moller CG, Davenport SL, et al. Usher syndrome: clinical findings and gene localization studies. Laryngoscope 1989;99:66-72.

9 Moller CG, Kimberling WJ, Davenport SL, et al. Usher syndrome: an otoneurologic study. Laryngoscope

10 Perbal B. Practical guide to molecular cloning. 2nd ed. New York: John Wiley, 1988

11 Weber JL. Informativeness of human (dC-dA)n.(dG-dT)n polymorphisms. Genomics 1990;7:524-30.

12 Dracopoli NC, O'Connell P, Elsner TI, et al. The CEPH consortium linkage map of human chromosome 1 . Genomics 1991;9:686-700.

13 Ott J. Analysis of human genetic linkage. Baltimore: Johns Hopkins University Press, 1991.

14 Heckenlively J. Retinitis pigmentosa. Philadelphia: Lippincott, 1988.

15 Gorlin RJ, Tilsner TJ, Feinstein S, et al. Usher's syndrome type III. Arch Otolaryngol 1979;105:353-4.

16 Karjalainen S, Terasvirta M, Karja J, Kaariainen H Usher's syndrome type III: ENG findings in four affected
and six unaffected siblings. $\mathcal{F}$ Laryngol Otol 1985;99:43-8.

17 Hallgren B. Retinitis pigmentosa combined with congenital deafness; with vestibulo-cerebellar ataxia and neural abnormality in a proportion of cases. Acta Psychiatr Scand (Suppl) 1959;138:1. 